\title{
Orientation-dependent ionization energies and interface dipoles in ordered molecular assemblies
}

Steffen Duhm ${ }^{1}$, Georg Heimel ${ }^{1} * *$, Ingo Salzmann ${ }^{1}$, Hendrik Glowatzki ${ }^{1}$, Robert L. Johnson ${ }^{2}$, Antje Vollmer ${ }^{3}$, Jürgen P. Rabe ${ }^{1}$, and Norbert Koch ${ }^{1}$

${ }^{1}$ Institut für Physik, Humboldt-Universität zu Berlin Newtonstr. 15, D-12489 Berlin, Germany

${ }^{2}$ Institut für Experimentalphysik, Universität Hamburg D-22761 Hamburg, Germany

${ }^{3}$ Berliner Elektronenspeicherring-Gesellschaft für Synchrotronstrahlung m.b.H. D-12489 Berlin, Germany

* E-mail:duhm@physik.hu-berlin.de

** E-mail: georg.heimel@physik.hu-berlin.de 
While an isolated individual molecule clearly has only one ionization potential, multiple values are found for molecules in ordered assemblies. Photoelectron spectroscopy of archetypical $\pi$-conjugated organic compounds on metal substrates combined with first-principles calculations and electrostatic modeling reveal the existence of a surface dipole built into molecular layers. Conceptually different from the surface dipole at metal surfaces, its origin lies in details of the molecular electronic structure and its magnitude depends on the orientation of molecules relative to the surface of an ordered assembly. Suitable pre-patterning of substrates to induce specific molecular orientations in subsequently grown films thus permits adjusting the ionization potential of one molecular species over up to $0.6 \mathrm{eV}$ via control over monolayer morphology. In addition to providing in-depth understanding of this phenomenon, our study offers design guidelines for improved organic/organic heterojunctions, hole- or electron-blocking layers, and reduced barriers for charge-carrier injection in organic electronic devices. 
It is well established that the work function $(\Phi)$ of metals depends on the crystal face ${ }^{1-3}$. $\Phi$ is defined as the energy difference between the Fermi level $\left(E_{F}\right)$ and the electrostatic potential above the sample, the vacuum level ( $\left.\mathrm{V}_{\mathrm{vac}}\right)$. For, e.g., copper, Фs of the (100), (110), and (111) surfaces are spread over a range of $0.5 \mathrm{eV}^{1,2}$. As $\mathrm{E}_{\mathrm{F}}$ is constant, this observation has been explained by the difference in the intrinsic "surface dipole": Differences in the geometric and, consequently, electronic structure cause a different amount of the electronic cloud to spill out of the bulk into the vacuum ${ }^{3,4}$. The resulting dipole raises $V_{\text {vac }}$ to a larger or smaller extent and thus impacts $\Phi^{4,5}$. Note that this effect can only be observed for laterally extended surfaces, as the spatial region above the sample where $V_{\text {vac }}$ is raised reaches farther away from the surface with increasing sample size (i.e., area of the exposed surface) ${ }^{6,7}$. Small metal clusters with multiple facets of different crystal orientations have only one well-defined work function ${ }^{8,9}$.

For van der Waals (i.e., non-covalent) crystals of non-dipolar molecules, surface dipoles and work-function anisotropy have not yet been explored ${ }^{6}$. While variations of the ionization potential (IP; the molecular equivalent of the work function) depending on the molecular orientation on a substrate have been reported before ${ }^{10-16}$, the prevalent interpretation in terms of variable photo-hole screening could never be satisfactorily quantified. Here, we propose a qualitatively different and novel explanation for the intriguing observation that one and the same molecule can have different - still welldefined - IPs if part of an ordered supramolecular structure. 
We performed X-ray photoelectron spectroscopy (XPS) and ultraviolet photoelectron spectroscopy (UPS) on $\alpha, \omega$-dihexyl-sexithiophene (DH6T) and $\alpha$ sexithiophene (6T), on Ag(111). The IPs of the molecules change by up to $0.6 \mathrm{eV}$ depending on whether they are lying down flat on the substrate or standing upright. In contrast to prior attempts ${ }^{10-16}$, we rationalize these observations in terms of the collective electrostatic effect of the highly anisotropic intra-molecular charge distribution based on density-functional theory (DFT) calculations and electrostatic modeling. Supplementary studies on different substrates and molecules underline the universality of the observed effects and their explanation. We stress that the general concept is valid also for single crystals and ordered polymers.

Since 6T and DH6T are used in organic field-effect transistors (OFETs) ${ }^{17-23}$, we discuss the immediate practical relevance of our findings in terms of the hole-injection barrier (HIB), a crucial parameter in organic electronic devices ${ }^{6,24,25}$. Pre-patterning an electrode with films of lying or standing DH6T allows for subsequent growth of films of likewise lying or standing 6T molecules and thus permits lowering the HIB at the Ag/6T contact by $0.4 \mathrm{eV}$. Furthermore, we derive conceptual guidelines for molecular design to optimize the energy-level alignment at inorganic/organic and organic/organic heterojunctions. Our findings thus open new routes towards organic electronic devices with improved performance and functionality, not only OFETs but also organic light emitting diodes (OLEDs) and organic solar cells. 
In general, the orientation of molecules in mono- and multilayers with respect to the substrate critically depends on the relative strengths of molecule-substrate interaction vs. intermolecular interaction $^{26,27}$. In the case of DH6T (and similar thiophene derivatives ${ }^{23}$ ), the molecules in the monolayer adsorb lying flat on metal surfaces ${ }^{28,29}$, whereas molecules in subsequent layers are "standing" with their long axis close to the surface normal $^{28,30}$. The experimental UPS spectra of DH6T on $\mathrm{Ag}(111)$ in the monolayer (L for "lying") and multilayer (S for "standing") regime are shown in Figure 1a, and Figure 1b displays the corresponding simulated spectra (vide infra). In the former, three low binding energy (BE) peaks can be clearly distinguished with their respective maxima at $1.6 \mathrm{eV}, 2.3 \mathrm{eV}$, and $2.9 \mathrm{eV}$ in the L-regime, and at $1.0 \mathrm{eV}, 1.7 \mathrm{eV}$, and $2.3 \mathrm{eV}$ in the Sregime. These peaks are derived from the highest occupied molecular orbital (HOMO), the HOMO-1, and the HOMO- $2^{31}$. All peaks are at $0.6 \mathrm{eV}$ lower $\mathrm{BE}$ for the (standing) multilayer (S) compared to the (lying) monolayer (L). Note that the change in the intensity ratio between the HOMO and HOMO-1 peak from 1:1 (L) to almost 1:2 (S) is indicative of the different orientation of the long molecular axes with respect to the surface normal due to photoemission selection rules ${ }^{31}$. As commonly observed ${ }^{6,24,32}$, the adsorption of a molecular monolayer leads to a decrease of $\Phi$. In our case, $\Delta \Phi \approx-0.7 \mathrm{eV}$ as determined from the secondary electron cutoff (SECO) (see Methodology section). This lowering of $\mathrm{V}_{\text {vac }}$ above the sample surface is often termed "interface dipole" (ID) ${ }^{6,24,32}$. No further reduction of $\Phi$ is observed upon subsequent deposition of the multilayer (Figure 1a). Consequently, the $-0.6 \mathrm{eV} \mathrm{BE}$ shift of the molecular levels directly translates into a reduction of the molecular IP (i.e., the energy difference between HOMO and $\mathrm{V}_{\mathrm{vac}}$ ) by this amount. In order to understand the physical origin of this shift, it is indispensable 
to investigate whether the core-levels are affected in the same way as the valence levels. For DH6T, the XPS spectra of the sulfur 2p core levels are shown in Figure 1c. While only one doublet ( $2 \mathrm{p}_{3 / 2}$ at $164.40 \mathrm{eV}$ and $2 \mathrm{p}_{1 / 2}$ at $\left.165.65 \mathrm{eV}\right)$ is observed in the L-regime, a second doublet appears in the S-regime which is shifted by $0.6 \mathrm{eV}$ towards lower BE. The consistency with the UPS data confirms that indeed all electronic states in the (lying) monolayer (L) are rigidly shifted to lower binding energy with respect to $\mathrm{E}_{\mathrm{F}}$ and $\mathrm{V}_{\mathrm{vac}}$.

In a second set of experiments, we investigate the orientation dependence of the IP in ordered layers of 6T. In contrast to DH6T, 6T on Ag(111) does not show an abrupt transition in morphology from the first to the second layer. The first 6T layers adsorb lying flat on $\operatorname{Ag}(111)^{31,33}$ and only a slow, gradual transition to almost standing molecules was suggested for very thick (> $200 \mathrm{~nm})$ films ${ }^{31}$, 33 . An exemplary UPS spectrum of $150 \AA$ $6 \mathrm{~T}$ (multilayer) on $\mathrm{Ag}(111)$ is shown in Figure 2a. Again, three peaks (HOMO, HOMO-1, and HOMO-2) can be identified with maxima at $1.8 \mathrm{eV}, 2.5 \mathrm{eV}$, and $3.1 \mathrm{eV}$. Since the $\pi$-electronic structure of $6 \mathrm{~T}$ is virtually identical to that of DH6T, the intensity ratio of the HOMO and HOMO-1 peaks of 1:1 is in accordance with the model of lying molecules ${ }^{31}$. Pre-patterning the $\operatorname{Ag}(111)$ substrate with a (lying) monolayer of DH6T and subsequent deposition of 6T does not change the valence spectrum of $6 \mathrm{~T}$ (Figure 2a). The ID between DH6T and 6T is negligible $(<0.1 \mathrm{eV})$ and thus indicative of vacuum-level alignment ${ }^{6,7}$ at this organic/organic heterojunction. In the next experiment, the Ag(111) surface was pre-patterned with a bilayer of DH6T, i.e., standing DH6T is now exposed on the surface. Deposition of $6 \mathrm{~T}$ onto this modified substrate significantly alters the UPS spectrum of 6T (Figure 2a). The valence levels are rigidly shifted by 0.4 
$\mathrm{eV}$ towards lower $\mathrm{BE}$ and the intensity ratio HOMO:HOMO-1 changes to 1:2. We therefore propose the growth model shown in Figure 3 for this organic heterostructure: Due to relatively strong $\pi-\pi$ interactions, 6T grows lying down (L) on the lying DH6T monolayer; on the second (standing) DH6T layer, 6T also grows standing upright (S) as $\pi-\pi$ interactions between $6 \mathrm{~T}$ molecules dominate over the interaction with the now inert surface composed of the alkyl chains of the underlying (standing) DH6T layer. As vacuum-level alignment ${ }^{6,7}$ prevails also between the bilayer DH6T and 6T, the shift of the HOMO observed in Figure 2a translates into a $0.4 \mathrm{eV}$ lower IP of $6 \mathrm{~T}$ in the Smorphology compared to the L-morphology. Building the same DH6T/6T heterostructure on polycrystalline gold instead of $\mathrm{Ag}(111)$ yields essentially the same results (see Supplementary Information), confirming that the observed effects are quite universal.

To understand our observations, it is important to consider that the kinetic energy of photoelectrons and thus the measured IP is affected by the polarization of neighboring matter by the photo-hole. For organic thin films on metals this includes (a) the metal substrate $^{6,7,24,34}$ and (b) surrounding molecules. With increasing thickness of the organic layer, the screening by the metal becomes less important for the topmost molecules (probed by XPS and UPS), resulting in an apparent shift of the molecular levels away from $\mathrm{V}_{\mathrm{vac}}$ and thus an increase of the measured IP. In our case, however, the IP decreases as multilayers of DH6T are deposited onto the first (lying) layer. The same is true for 6T deposited on a bilayer of DH6T, where the IP also decreases compared to direct deposition onto $\mathrm{Ag}(111)$ or onto a (lying) monolayer of DH6T. We conclude that 
screening of the photo-hole by the metal can not account for the observed lowering of the molecular IP.

It may be speculated, however, that the photo-hole is more efficiently screened by surrounding standing molecules than by surrounding flat-lying molecules and, for similar organic compounds, the impact of molecular orientation on the IP has indeed been qualitatively rationalized in terms of the polarization energy depending on the packing density and/or morphology ${ }^{10,11,15,16}$. Here, we provide an upper limit for this proposed variation in polarization energy: A molecule in the topmost organic layer is surrounded by the metal substrate (at some distance), neighboring molecules in the half-space below, and by vacuum in the half-space above; a molecule deeper in the organic layer is additionally surrounded by molecules on top. ${ }^{35}$ Clearly, the presence or absence of neighboring molecules in the upper half-space must have a stronger effect on the polarization energy (and thus the measured IP) than differences in the orientation of neighboring molecules. Re-examining the XPS data in Figure 1c, we find that the binding energy of the $S(2 p)$ peaks attributed to the first (lying) layer of DH6T does not change upon deposition of subsequent layers of DH6T. In order to further confirm our reasoning, we performed additional independent measurements (see Supplementary Information) which yield an upper limit of $0.15 \mathrm{eV}$ for the difference in polarization energy between bulk and surface. ${ }^{36}$ We thus conclude that the $0.6 \mathrm{eV}[0.4 \mathrm{eV}]$ difference in the IP between standing and lying DH6T [6T] can not be explained in terms of photo-hole screening effects alone, and that another mechanism must be involved. 
In order to understand the remarkable finding of one molecule having a different IP depending on its orientation in an ordered supramolecular structure ${ }^{10-16,28}$, we performed plane-wave based DFT calculations employing periodic boundary conditions and the repeated-slab approach on single layers of standing and lying DH6T and 6T molecules based on available structural data (see Methodology section for details) ${ }^{13,29,37-}$ 39. The occupied density-of-states (DOS) for a lying (L) and standing (S) layer of DH6T is shown in Figure 1b. In addition to good qualitative agreement with the experimental UPS spectra (Figure 1a), we find that indeed, all molecular levels are closer to $\mathrm{V}_{\mathrm{vac}}$ for the S-layer compared to the L-layer, i.e., the IP is lower for standing molecules. For 6T, DFT calculations yield similar results: the molecular levels are closer to $\mathrm{V}_{\mathrm{vac}}$ for the $\mathrm{S}$ layer, i.e., the IP is again lower compared to molecules in the L-layer. The corresponding DOS is shown in Figure 2b.

To rationalize the fundamental mechanism that gives rise to this shift, we consider electrostatics on the molecular scale in analogy to the situation for extended metal surfaces vs. metal nanoclusters (vide supra). In Figure 4a we show the electrostatic potential (obtained from DFT calculations) around one isolated 6T molecule relative to its HOMO energy. The coloring scheme (together with the energy scale) thus indicates the amount of work required to promote one electron out of the HOMO to any given point in space. Consistent with the observation that an isolated molecule has only one well-defined $\mathrm{IP}^{40}$, the potential converges to a single value of $\mathrm{V}_{\mathrm{vac}}$ (cyan) in any direction on a sub-molecular length scale. It becomes apparent though, that $\mathrm{V}_{\mathrm{vac}}$ is higher directly above the (negatively charged) $\pi$-electron system (blue region marked "L") than next to 
the hydrogen-terminated ends of the molecules (green region marked "S"). To a first approximation, the potential distributions of the individual molecules add up as molecules assemble into, e.g., crystals or layers ${ }^{6}$. For molecules standing in a layer, the Sregion dominates the electrostatic potential above the layer while for lying molecules the L-region determines the value of $\mathrm{V}_{\text {vac }}$ above the layer. This is shown in Figure $4 \mathrm{~b}$ and $4 \mathrm{c}$, where the electrostatic potential of the molecular layers is plotted relative to their respective $\mathrm{HOMO}$ energies. Clearly, $\mathrm{V}_{\mathrm{vac}}$ is higher (blue) above the layer with lying molecules than it is above the layer comprising only standing molecules (green), thus leading to the lower IP of the latter. To confirm the validity of our model, we performed DFT calculations also for lying and standing layers of pentacene, where the difference between the two respective IPs was experimentally determined to be $c a .0 .5 \mathrm{eV}$ and no satisfactory explanation could be found ${ }^{11,13,14}$. In good agreement, our calculations yield a difference of $0.6 \mathrm{eV}$.

While DFT calculations permit quantitative analysis, we offer an even simpler, purely electrostatic model in order to establish a more intuitive picture. We approximate the charge distribution corresponding to one 6T molecule (Figure 5a) by a number of point-charges (Figure 5b): The $\pi$-electron system above and below each ring is clearly negatively charged; this is represented by negative point charges of $-0.5 e$ (elementary charge) placed 0.5 bohr above and below the molecular plane. These negative charges are compensated by a $+1.0 e$ point charge in the plane of the molecule. This pattern is repeated six times (one for each ring) along the long molecular axis with a spacing of 8 bohr ( $\approx$ distance between individual thiophene rings). From this model-molecule, a 2D 
(see Methodology section) molecular crystal is built (Figure 5c), consisting of 21 standing model-molecules in one layer with a distance of 6 bohr between their long molecular axes. Below that, one more such layer is placed with a gap of 10 bohr to the first layer.

This model-crystal has two distinctly different crystal faces: one (top and bottom) is terminated by the point-charge pattern $-0.5|+1.0|-0.5||-0.5|+1.0|-0.5|| \ldots$ that represents the hydrogen-terminated ends of the 6T molecules exposed in a standing layer; the other (left and right) is terminated by negatively charged sheets that represent the $\pi$-electron cloud exposed in a lying layer. The electrostatic potentials all point charges are summed up to yield the potential within and around the model-crystal (shown in Figure 5c). In analogy to the results from the DFT calculations, we find an extended region of lower electrostatic potential (green) over the hydrogen-terminated ends of the molecules, relevant for the standing layer (S). Above the $\pi$-system, there is an extended region of higher electrostatic potential (cyan), relevant for the lying layer (L). As a consequence, the work required to promote an electron from any one energy level within the modelcrystal [e.g., the HOMO or the $\mathrm{S}(2 \mathrm{p})$ core levels] into the spatial region above the hydrogen-terminated ends (S) is less than that for promoting an electron into the spatial region above the $\pi$-electron clouds $(\mathrm{L})$. This difference can be measured as soon as the lateral extent of the supramolecular structure is large compared to a single molecule. The situation is equivalent to the presence of a considerable intrinsic surface dipole $e^{4,5}$ (negatively charged $\pi$-system, positively charged molecular plane below) at the surface of the lying molecular layers whereas no such dipole occurs at the surface of standing 
layers. Note that, while the surface dipole of metals is "pushed back" upon adsorption of molecules (thus giving rise to an ID) ${ }^{6,24,25,41,42}$, the intra-molecular surface dipole remains unaffected by establishing contact to either the (metal) substrate or another organic layer. Our choice for the amount and separation of the charges in the modelmolecule and -crystal is justified a posteriori as the difference in $V_{\text {vac }}$ above the $\pi$-system (L) and hydrogen-terminated ends (S) amounts to $0.4 \mathrm{eV}$ (Figure 5c) which compares favorably to the experimentally observed differences in the IP of standing vs. lying $6 \mathrm{~T}$ layers (Figure 2a). Our results are summarized in the energy-level diagram in Figure 6.

We emphasize that the presence of an intrinsic surface dipole in molecular layers has important implications for organic electronics: Figure $6 \mathrm{~b}$ shows that an electronic heterojunction with a large energy-level offset can, in fact, be realized with only one molecular species (DH6T), a so far unexplored concept. As such heterojunctions play a crucial role in organic solar cells and in the context of hole- or electron blocking layers in OLEDs, we suggest that this energy-level offset may be tuned by chemically tailoring the end-groups on the $\pi$-conjugated core (alkyl segments in DH6T). Inserting a dipole pointing away from the core by, for example, introducing heteroatoms or making them more electron withdrawing (e.g., by fluorination ${ }^{23}$ ), increases the IP of the standing layer (thus decreasing the offset); a dipole pointing towards the core or more electron-donating groups can be expected to further decrease the IP of the S-layer (thus increasing the offset). As it determines the barrier for charge-carrier injection into the organic, also the energy difference between the Fermi level of a (metallic) electrode and the conducting states in the active organic layer is of uttermost importance for optimizing the 
performance of organic electronic devices ${ }^{6,24,25}$. For the occupied manifold of states discussed in the present study, this is the hole injection barrier (HIB) ${ }^{6,24}$. While control over its orientation already allows considerably reducing the HIB into one and the same molecule by several tenths of an $\mathrm{eV}$, the strategies for chemical modification suggested above can be expected to contribute to a further lowering; similar considerations hold for the unoccupied manifold of states connected to the electron injection barrier in n-type OFETs $^{23}$.

As also other important factors in organic electronic devices, e.g., photoluminescence or charge-carrier mobility ${ }^{17,43,44}$, depend on the orientation of the (intrinsically anisotropic) molecules, our approach of pre-patterning a metal surface with appropriate molecular species (shown here for DH6T) seems to be a promising tool for controlling the orientation of subsequently deposited molecules; for 6T, the gradual transition $^{31,33}$ from lying to standing orientation can be reduced from hundreds of layers to only two.

\section{Methodology}

UPS experiments were performed at the FLIPPER II end-station at HASYLAB (Hamburg, Germany) ${ }^{45}$. The interconnected sample preparation chambers (base pressure $2 \times 10^{-9}$ mbar) and analysis chamber (base pressure $2 \times 10^{-10}$ mbar) allowed sample transfer without breaking ultrahigh vacuum (UHV) conditions. The Ag(111) single crystal was cleaned by repeated Ar-ion sputtering and annealing cycles (up to $550{ }^{\circ} \mathrm{C}$ ). DH6T (H. C. 
Starck GmbH) and 6T (Aldrich) were evaporated using resistively heated pinhole sources, at evaporation rates of about $1 \AA / \mathrm{min}$. The film mass-thickness was monitored with a quartz crystal microbalance. Spectra were recorded with a double-pass cylindrical mirror analyzer with an energy resolution of $150 \mathrm{meV}$ and a photon energy of $22 \mathrm{eV}$. The SECOs were measured with the sample biased at $-3.00 \mathrm{~V}$. The work function was calculated by subtracting the sum of total width (i.e., SECO to Fermi level) of the photoelectron spectrum and bias voltage from the photon energy. XPS experiments were performed at the end-station SurICat (beam line PM4) at the BESSY synchrotron (Berlin, Germany) ${ }^{46}$. There, the UHV system consists of interconnected sample preparation (base pressure $1 \times 10^{-8} \mathrm{mbar}$ ) and analysis (base pressure $1 \times 10^{-10} \mathrm{mbar}$ ) chambers. The spectra were collected with a hemispherical electron energy analyzer (Scienta SES 100) with 120 meV energy resolution at $20 \mathrm{eV}$ pass energy. The photon energy was $400 \mathrm{eV}$. Additional XPS spectra were measured with an $\mathrm{Al} \mathrm{K}_{\alpha 1 / 2}$ lab source in a custom UHV system. Sample preparation for XPS measurements was analogous to that for UPS measurements. All preparation steps and measurements were performed at room temperature. The fitting of UPS and XPS spectra (Voigt peaks and Shirley background) was performed with the program WINSPEC (Namur University).

In the DFT calculations the repeated slab approach was employed with the vacuum region separating two consecutive molecular layers being $\geq 20 \AA$. The PW91 exchange-correlation functional was used. For the valence-core interactions, the projector augmented-wave method ${ }^{47}$ was employed permitting the low kinetic energy cutoff of 20 Ryd for the plane-wave expansion of the valence Kohn-Sham orbitals. Monkhorst-Pack 
grids of $1 \times 4$ k-points (lying 6T and lying pentacene), $1 \times 2$ (lying DH6T), and $4 \times 4$ (all standing structures) were used for the integration of the 2D Brillouin zone. The isolated $6 \mathrm{~T}$ molecule was calculated in a $50 \times 30 \times 20 \AA$ box at the $\Gamma$-point only. The atomic positions within the molecules were optimized until all remaining forces were $\leq 0.01$ eV/Å. All calculations were performed with the VASP $\operatorname{code}^{48,49}$. The 3D graphics were produced with XCrysDen ${ }^{50}$.

In the absence of experimental structural data for (lying) monolayers on Ag(111), the surface unit cells of the respective molecules on $\mathrm{Au}(111)$ were used in the DFT calculations: $a=25 \AA, b=6 \AA, \gamma=65.0^{\circ}$ for $6 \mathrm{~T}^{29}$; $\mathrm{a}=38 \AA, \mathrm{b}=16 \AA, \gamma=19.0^{\circ}$ for $\mathrm{DH}^{2} \mathrm{~T}^{29}$; and $\mathrm{a}=5.76 \AA, \mathrm{b}=15.3 \AA, \gamma=79.1^{\circ}$ for pentacene ${ }^{13,37}$. Since also the actual thin film structures for the (standing) multilayer systems are not known from experiment, a single layer of standing molecules was cut out of the respective bulk structures for the DFT calculations; the lateral unit cells containing two molecules arranged in typical herringbone fashion were taken to be $a=7.851 \AA, b=6.029 \AA, \gamma=0^{\circ}$ for $6 T^{38}$ and $a=6.266 \AA, b=7.742 \AA$, $\gamma=84.68^{\circ}$ for pentacene ${ }^{13,39}$; the long molecular axes in these structures are tilted by ca $25^{\circ}$ from the layer normal. For DH6T, a single layer of $6 \mathrm{~T}$ was cut out of the $6 \mathrm{~T}$ bulk structure and a hexyl chain was manually attached to either end of the molecules; in the course of the subsequent DFT calculations, the geometry of these hexyl chains was optimized.

Due to intrinsic shortcomings of DFT, the calculated HOMO energies usually underestimate IPs. As photo-hole screening is not included in standard DFT calculations, 
our calculated shifts in IP have to be regarded as shifts of the initial electronic states prior to removal of the photo-electron. We attribute the overestimation of the shifts in IP to the high degree of order and uniformity in the simulations (not necessarily present in experiment) and to possible discrepancies between the structures assumed for the calculations and the actual structures probed in experiment.

The two-dimensional model molecular crystal in Figure 6c is equivalent to a three-dimensional crystal where the point-charge pattern shown in Figure 6c is infinitely continued into and out of the plane of drawing. Note that in the case of two-dimensional electrostatics, the potential, $V$, decreases with increasing distance, $r$, from a point charge as $V \propto-\ln \left(r^{2}\right)$ instead of the familiar $V \propto 1 / r$ for the three-dimensional case.

\section{Acknowledgements}

The authors thank H. C. Starck GmbH for providing DH6T. NK acknowledges financial support by the Emmy Noether Program (DFG). GH is a Marie-Curie Fellow under the INSANE project (contract no. 021511). We thank L. Romaner for helpful discussions and the SFB 488 "Mesoscopically Organized Composites" for financial support. 


\section{References}

1. Michaelson, H. B. The work function of the elements and its periodicity. J. Appl. Phys. 48, 4729-4733 (1977).

2. Skriver, H. L. \& Rosengaard, N. M. Surface energy and work function of elemental metals. Phys. Rev. B 46, 7157-7168 (1992).

3. Smoluchowski, R. Anisotropy of the Electronic Work Function of Metals. Phys. Rev. 60, 661-674 (1941).

4. Lang, N. D. \& Kohn, W. Theory of Metal Surfaces: Work Function. Phys. Rev. B 3, 1215-1223 (1971).

5. Weinert, M. \& Watson, R. E. Contributions to the work function of crystals. Phys. Rev. B 29, 3001-3008 (1984).

6. Ishii, H., Sugiyama, K., Ito, E. \& Seki, K. Energy Level Alignment and Interfacial Electronic Structures at Organic/Metal and Organic/Organic Interfaces. Adv. Mater. 11, 605-625 (1999).

7. Cahen, D. \& Kahn, A. Electron Energetics at Surfaces and Interfaces: Concepts and Experiments. Adv. Mater. 15, 271-277 (2003).

8. Häkkinen, H. et al. On the Electronic and Atomic Structures of Small $\mathrm{Au}_{N}{ }^{-}(N=4-$ 14) clusters: A Photoelectron Spectroscopy and Density-Functional Study. J. Phys. Chem. A 107, 6168-6175 (2003).

9. Li, J., Li, X., Zhai, H. J. \& Wang, L. S. Au 20 : A Tetrahedral Cluster. Science 299, 864-867 (2003). 
10. Friedlein, R. et al. High intercalation levels in lithium perylene stoichiometric compounds. Chem. Phys. Lett. 354, 389-394 (2002).

11. Fukagawa, H. et al. Origin of the highest occupied band position in pentacene films from ultraviolet photoelectron spectroscopy: Hole stabilization versus band dispersion. Phys. Rev. B 73, 245310 (2006).

12. Ivanco, J., Winter, B., Netzer, T. R. \& Ramsey, M. G. Substrate-Mediated Electronic Structure and Properties of Sexiphenyl Films. Adv. Mater. 15, 18121815 (2003).

13. Koch, N. et al. Molecular orientation dependent energy levels at interfaces with pentacene and pentacenequinone. Org. Electron. 7, 537-545 (2006).

14. Ihm, K. et al. Molecular orientation dependence of hole-injection barrier in pentacene thin film on the Au surface in organic thin film transistor. Appl. Phys. Lett. 89, 033504 (2006).

15. Ivanco, J. et al. Sexithiophene films on ordered and disordered $\mathrm{TiO}_{2}(110)$ surfaces: Electronic, structural and morphological properties. Surf. Sci. 601, 178187 (2007).

16. Ivanco, J., Netzer, F. P. \& Ramsey, M. G. On validity of the Schottky-Mott rule in organic semiconductors: Sexithiophene on various substrates. J. Appl. Phys. 101, 103712 (2007).

17. Dinelli, F. et al. Spatially Correlated Charge Transport in Organic Thin Film Transistors. Phys. Rev. Lett. 92, 116802 (2004).

18. Dodabalapur, A., Torsi, L. \& Katz, H. E. Organic Transistors: Two-Dimensional Transport and Improved Electrical Characteristics. Science 268, 270-271 (1995). 
19. Loi, M. A. et al. Supramolecular organization in ultra-thin films of $\alpha$ sexithiophene on silicon dioxide. Nat. Mater. 4, 81-85 (2005).

20. Facchetti, A. et al. Building Blocks for n-Type Molecular and Polymeric Electronics. Perfluoroalkyl- versus Alkyl-functionalized Oligothiophenes (nT; n=2-6). Systematics of Thin Film Microstructure, Semiconductor Performance, and Modeling of Majority Charge Injection in Field-Effect Transistors. J. Am. Chem. Soc. 126, 13859-13874 (2004).

21. Garnier, F. et al. Molecular Engineering of Organic Semiconductors: Design of Self-Assembly Properties in Conjugated Thiophene Oligomers. J. Am. Chem. Soc. 115, 8716-8721 (1993).

22. Halik, M. et al. Relationship Between Molecular Structure and Electrical Performance of Oligothiophene Organic Thin Film Transistors. Adv. Mater. 15, 917-922 (2003).

23. Dholakia, G. R., Meyyappan, M., Facchetti, A. \& Marks, T. J. Monolayer to Multilayer Nanostructural Growth Transition in N-Type Oligothiophenes on Au(111) and Implications for Organic Field-Effect Transistor Performance. Nano Lett. 6, 2447-2455 (2006).

24. Kahn, A., Koch, N. \& Gao, W. Y. Electronic Structure and Electrical Properties of Interfaces between Metals and $\pi$-Conjugated Molecular Films. Journal of Polymer Science, Part B: Polymer Physics 41, 2529-2548 (2003).

25. Koch, N., Elschner, A., Schwartz, J. \& Kahn, A. Organic molecular films on gold versus conducting polymer: Influence of injection barrier height and morphology on current-voltage characteristics. Appl. Phys. Lett. 82, 2281-2283 (2003). 
26. Hooks, D. E., Fritz, T. \& Ward, M. D. Epitaxy and Molecular Organization on Solid Substrates. Adv. Mater. 13, 227-241 (2001).

27. Witte, G. \& Wöll, C. Growth of aromatic molecules on solid substrates for applications in organic electronics. J. Mater. Res. 19, 1889-1916 (2004).

28. Duhm, S., Glowatzki, H., Rabe, J. P., Koch, N. \& Johnson, R. L. Influence of alkyl chain substitution on sexithienyl-metal interface morphology and energetics. Appl. Phys. Lett. 88, 203109 (2006).

29. Glowatzki, H., Duhm, S., Braun, K.-F., Rabe, J. P. \& Koch, N. Molecular chains and carpets of sexithiophenes on Au(111). Phys. Rev. B 76, 125425 (2007).

30. Duhm, S. et al. Vacuum sublimed $\alpha, \omega$-dihexylsexithiophene thin films: Electronic structure and molecular orientation. in preparation (2007).

31. Heiner, C. E. et al. Anisotropy in ordered sexithiophene thin films studied by angle-resolved photoemission using combined laser and synchrotron radiation. Appl. Phys. Lett. 87, 093501 (2005).

32. Lang, N. D. \& Norskov, J. K. Interaction of helium with a metal surface. Phys. Rev. B 27, 4612-4616 (1983).

33. Yoshikawa, G., Kiguchi, M., Ikeda, S. \& Saiki, K. Molecular orientations and adsorption structures of $\alpha$-sexithienyl thin films grown on $\mathrm{Ag}(110)$ and $\mathrm{Ag}(111)$ surfaces. Surf. Sci. 559, 77-84 (2004).

34. Koch, N. et al. Influence of molecular conformation on organic/metal interface energetics. Chemical Physics Letters 413, 390-395 (2005).

35. Hill, I. G., Mäkinen, A. J. \& Kafafi, Z. H. Initial stages of metal/organic semiconductor interface formation. J. Appl. Phys. 88, 889-895 (2000). 
36. Salaneck, W. R. Intermolecular Relaxation Energies in Anthracene. Phys. Rev. Lett. 40, 60-63 (1978).

37. France, C. B., Schroeder, P. G., Forsythe, J. C. \& Parkinson, B. A. Scanning Tunneling Microscopy Study of the Coverage-Dependent Structures of Pentacene on Au(111). Langmuir 19, 1274-1281 (2003).

38. Horowitz, G. et al. Growth and Characterization of Sexithiophene Single Crystals. Chem. Mater. 7, 1337-1341 (1995).

39. Mattheus, C. C. et al. Polymorphism in pentacene. Acta Crystallogr., Sect. C 57, 939-941 (2001).

40. Coropceanu, V. et al. Hole- and Electron-Vibrational Couplings in Oligoacene Crystals: Intramolecular Contributions. Phys. Rev. Lett. 89, 275501 (2002).

41. Ito, E. et al. Interfacial electronic structure of long-chain alkane/metal systems studied by UV-photoelectron and metastable atom electron spectroscopies. Chem. Phys. Lett. 287, 137-142 (1998).

42. Witte, G., Lukas, S., Bagus, P. S. \& Wöll, C. Vacuum level alignment at organic/metal junctions: "Cushion" effect and the interface dipole. Appl. Phys. Lett. 87, 263502 (2005).

43. Sreearunothai, P. et al. Influence of Copolymer Interface Orientation on the Optical Emission of Polymeric Semiconductor Heterojunctions. Phys. Rev. Lett. 96, 117403 (2006).

44. Sirringhaus, H. et al. Two-dimensional charge transport in self-organized, highmobility conjugated polymers. Nature 401, 685-688 (1999). 
45. Johnson, R. L. \& Reichardt, J. FLIPPER II - a new photoemission system in HASYLAB. Nucl. Instr. Meth. Phys. Res. 208, 791-796 (1983).

46. Vollmer, A. et al. The effect of oxygen exposure on pentacene electronic structure. Eur. J. Phys. E 17, 339-343 (2005).

47. Kresse, G. \& Joubert, D. From ultrasoft pseudopotentials to the projector augmented-wave method. Phys. Rev. B 59, 1758-1775 (1999).

48. Kresse, G. \& Furthmüller, J. Efficiency of ab-initio total energy calculations for metals and semiconductors using a plane-wave basis set. Comp. Mater. Sci. 6, 1550 (1996).

49. Kresse, G. \& Furthmüller, J. Efficient iterative schemes for $a b$ initio total-energy calculations using a plane-wave basis set. Phys. Rev. B 54, 11169-11186 (1996).

50. Kokalj, A. Computer graphics and graphical user interfaces as tools in simulations of matter at the atomic scale. Comp. Mater. Sci. 28, 155-168 (2003). 


\section{Figure Captions}

\section{Figure 1 Experimental and theoretical photoelectron spectra of DH6T.}

a, Experimental UPS spectra showing the valence region of $4 \AA$ of DH6T (purple), and $40 \AA$ of DH6T (cyan) on Ag(111), corresponding to a lying monolayer (L) and a second standing layer (S). The dotted lines indicate the least-squares fit to determine the peak positions. The inset shows the secondary electron cut-offs used to determine the work function of the respective sample. $\mathbf{b}$, DFT calculated density-of-states (DOS) of a single layer of lying (purple) and standing (cyan) DH6T molecules; the origin of the energy scale is the respective vacuum level. The inset shows the chemical structure of DH6T. c, Experimental XPS spectra in the sulfur 2p region for $4 \AA$ (purple) and $30 \AA$ (cyan) of DH6T on Ag(111); these coverages correspond to approximately a lying monolayer (L) and a second standing layer (S), respectively.

\section{Figure 2 Experimental and theoretical photoelectron spectra of 6T.}

a, Experimental UPS spectra showing the valence region of $150 \AA$ of $6 \mathrm{~T}$ (dashed red), $100 \AA$ of $6 \mathrm{~T}$ on $4 \AA$ of DH6T (solid red) and $50 \AA$ of $6 \mathrm{~T}$ on $40 \AA$ of DH6T (solid green) with $\mathrm{Ag}(111)$ as substrate in all cases, corresponding to lying (L) and standing (S) 6T structures. The dotted lines indicate the least-squares fit to determine the peak positions. The inset shows the secondary electron cut-offs used to determine the work function of the respective sample. b, DFT calculated density-of-states (DOS) of a single layer of lying (red) and standing (green) 6T molecules; the origin of the energy scale is the respective vacuum level. The inset shows the chemical structure of $6 \mathrm{~T}$. 


\section{Figure 3 Growth model for 6T/DH6T heterostructures on Ag(111).}

a, On clean $\mathrm{Ag}(111)$ and on a monolayer of lying DH6T (cyan), 6T (green) grows with its long molecular axes parallel to the surface. b, On a standing layer of DH6T (purple), $6 \mathrm{~T}$ (red) grows with its long molecular axes inclined to the surface normal.

\section{Figure 4 Electrostatic potential for an isolated $6 \mathrm{~T}$ molecule and $6 \mathrm{~T}$ layers.}

a, Electrostatic potential around a single 6T molecule within the molecular $(x-z)$ plane and perpendicular $(x-y)$ to the molecular plane. Regions of higher potential $(L)$ are found over the $\pi$-electron systems (blue), while regions of lower potential (S) are located around the hydrogen termination of the molecule (green). b, Electrostatic potential of a single layer of standing $6 \mathrm{~T}$ molecules. c, Electrostatic potential of a single layer of lying $6 \mathrm{~T}$ molecules. The origin of the energy scale in each plot is the respective HOMO energy. The colors in the vacuum above the standing layer (green), b, and the lying layer (blue), c, thus indicate that the IP of the former is smaller than that of the latter (see color bars).

\section{Figure 5 Electrostatic modeling of the orientation-dependent ionization potential.}

To approximate the electron density of a single $6 \mathrm{~T}$ molecule, a, a series of point charges, b, is used. Distances and charges are given in atomic units. c, Electrostatic potential in and around a model molecular crystal consisting of two layers of 21 model-molecules, $\mathbf{b}$, each. See text for details. 


\section{Figure 6 Energy-level diagram for the 6T/DH6T heterostructures on Ag(111).}

a, Energy-level diagram for the lying-6T|lying-DH6T-monolayer|Ag(111) structure. b, Energy-level diagram for the standing-6T|standing-DH6T|lying-DH6Tmonolayer|Ag(111) structure. The ionization potential (IP), work function (Ф), and hole injection barrier (HIB) are given in $\mathrm{eV}$. The vertical lines indicate the vacuum level ( $\left.\mathrm{V}_{\text {vac }}\right)$, the Fermi energy $\left(\mathrm{E}_{\mathrm{F}}\right)$, and the highest occupied molecular orbitals (HOMO). The onset of the HOMO peaks is approx. $0.5 \mathrm{eV}$ closer to $\mathrm{E}_{\mathrm{F}}$ than the peak maxima listed here. The intrinsic surface dipole due to the $\pi$-electron clouds over the molecular planes in the lying layer(s) is highlighted in red. The work function of the pristine $\operatorname{Ag}(111)$ surface $(=4.4 \mathrm{eV})$ as well as the interface dipole $(=0.7 \mathrm{eV})$ are also shown. 
Figure 1

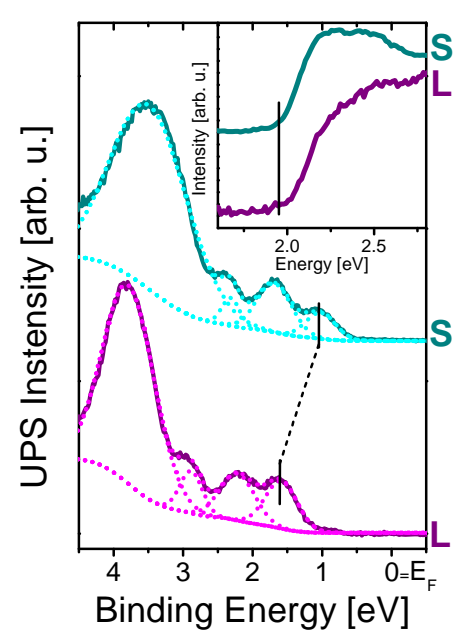

(a)

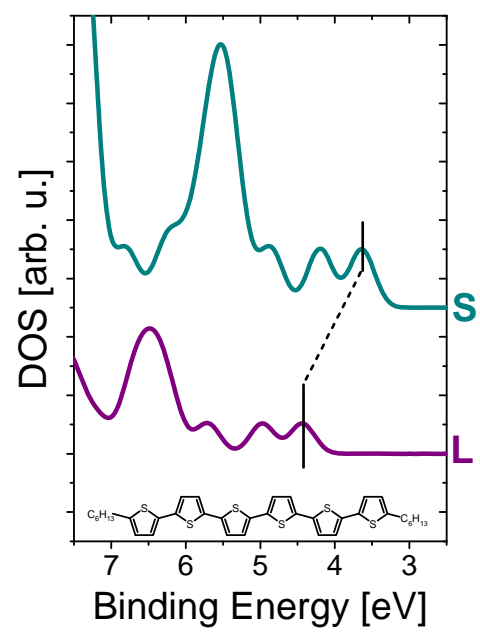

(b)

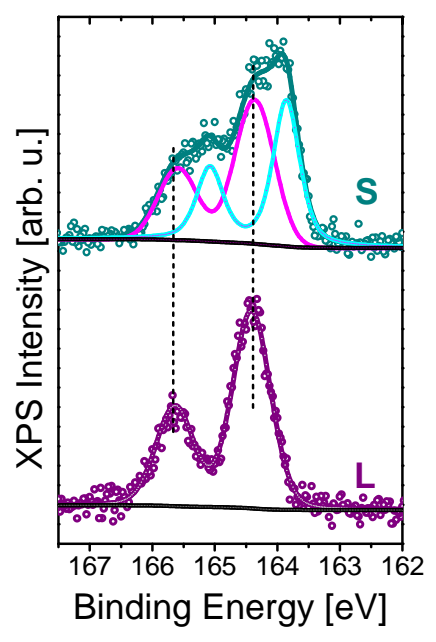

(c) 
Figure 2

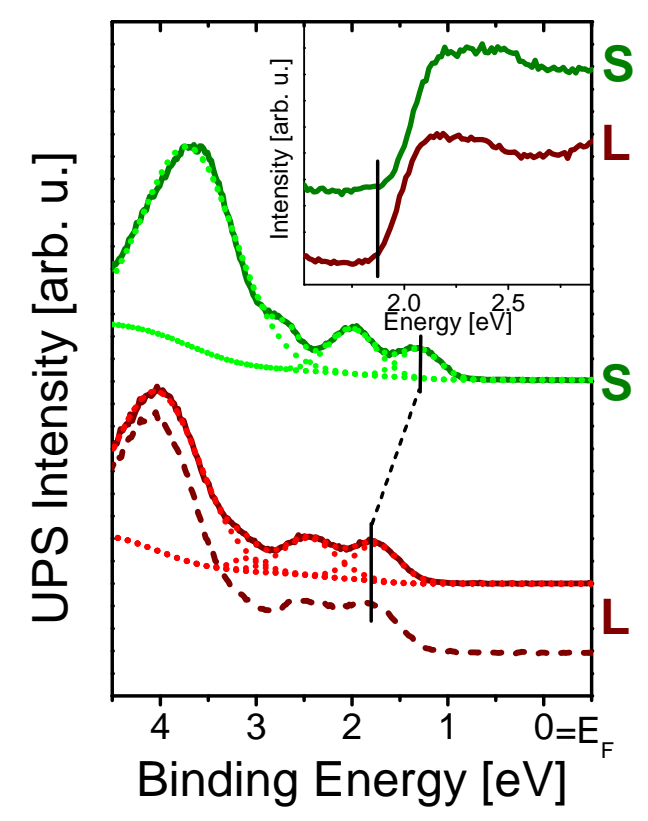

(a)

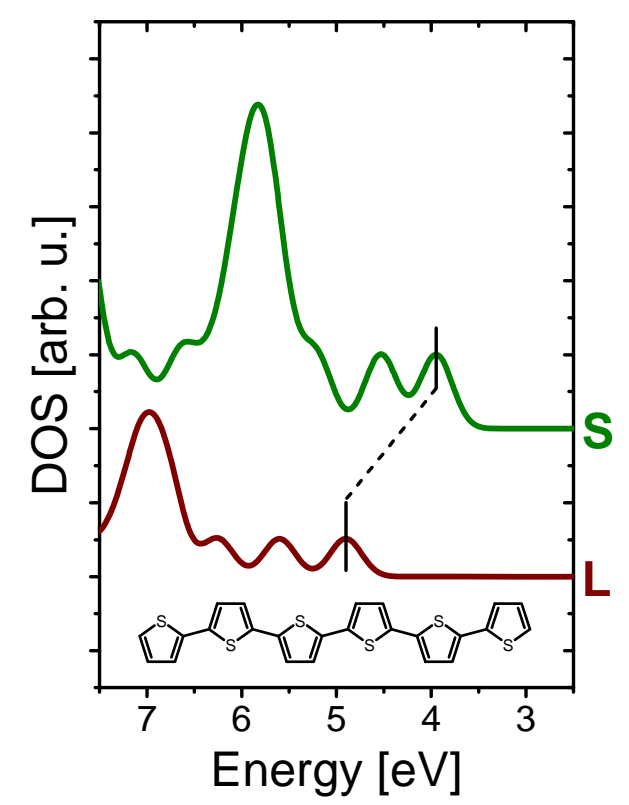

(b) 
S. Duhm et al., Nature Materials, accepted

\section{Figure 3}

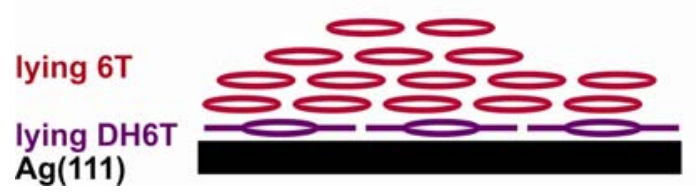

(a)

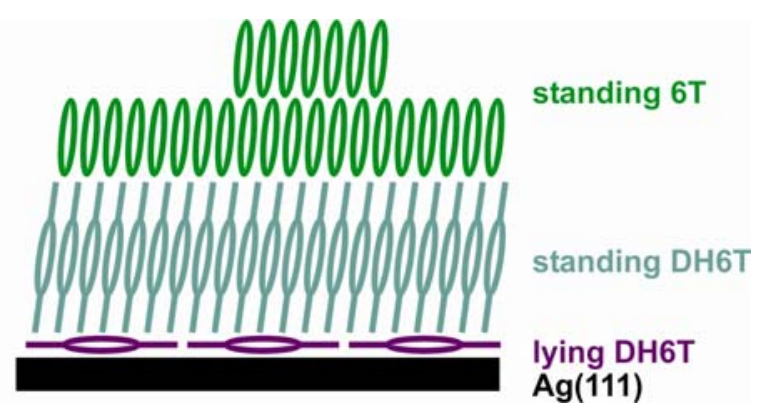

(b) 
S. Duhm et al., Nature Materials, accepted

\section{Figure 4}

(a)

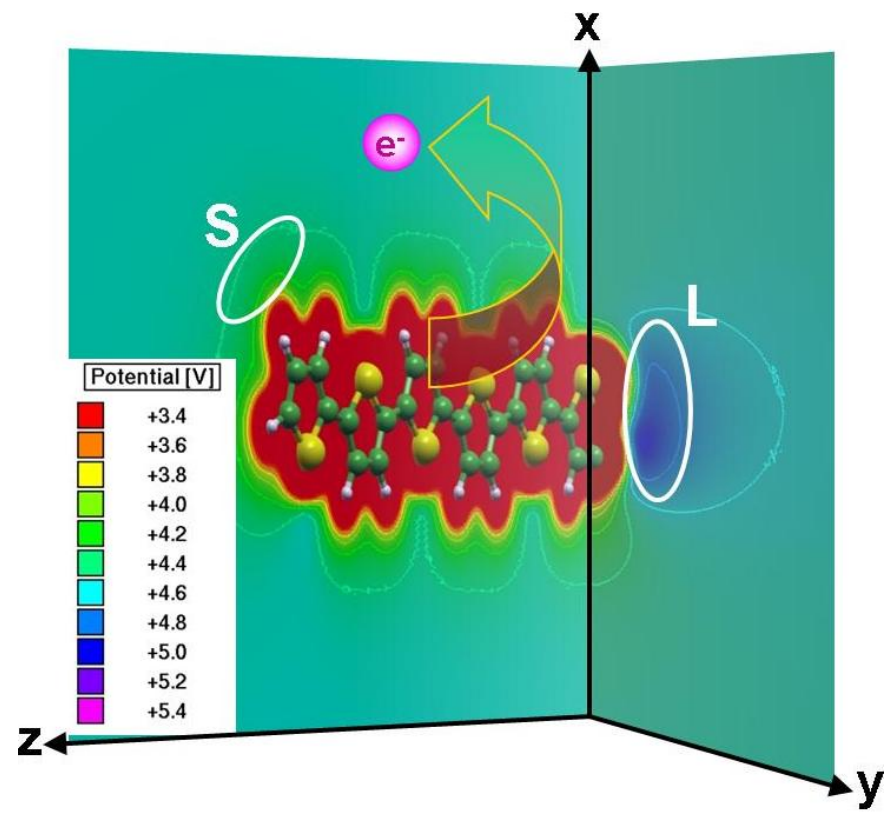

(b)

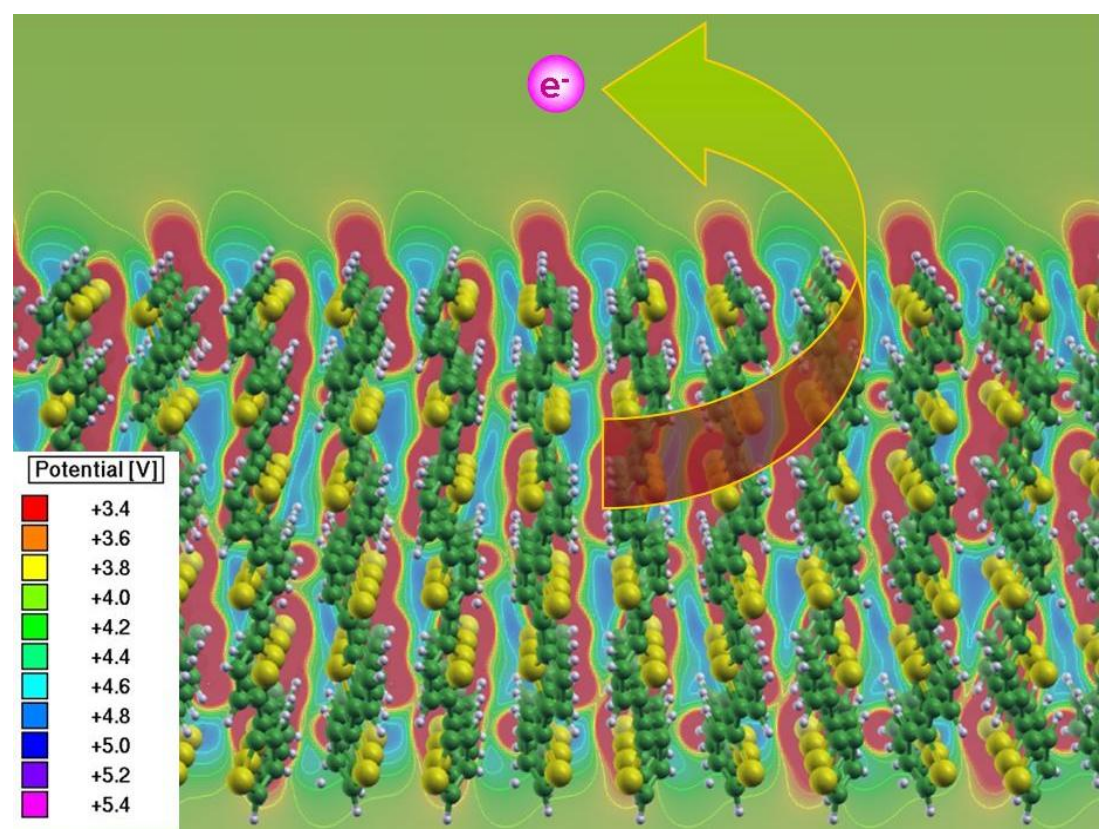

(c)

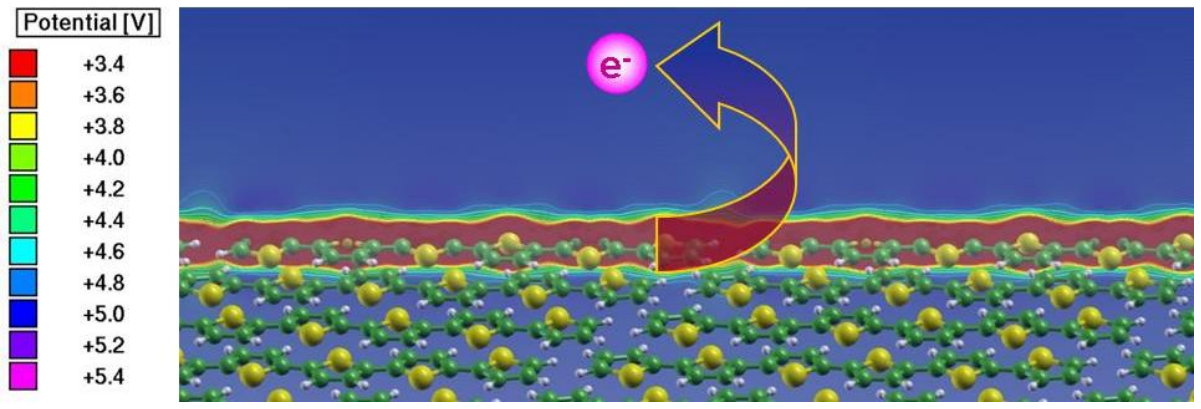


S. Duhm et al., Nature Materials, accepted

\section{Figure 5}
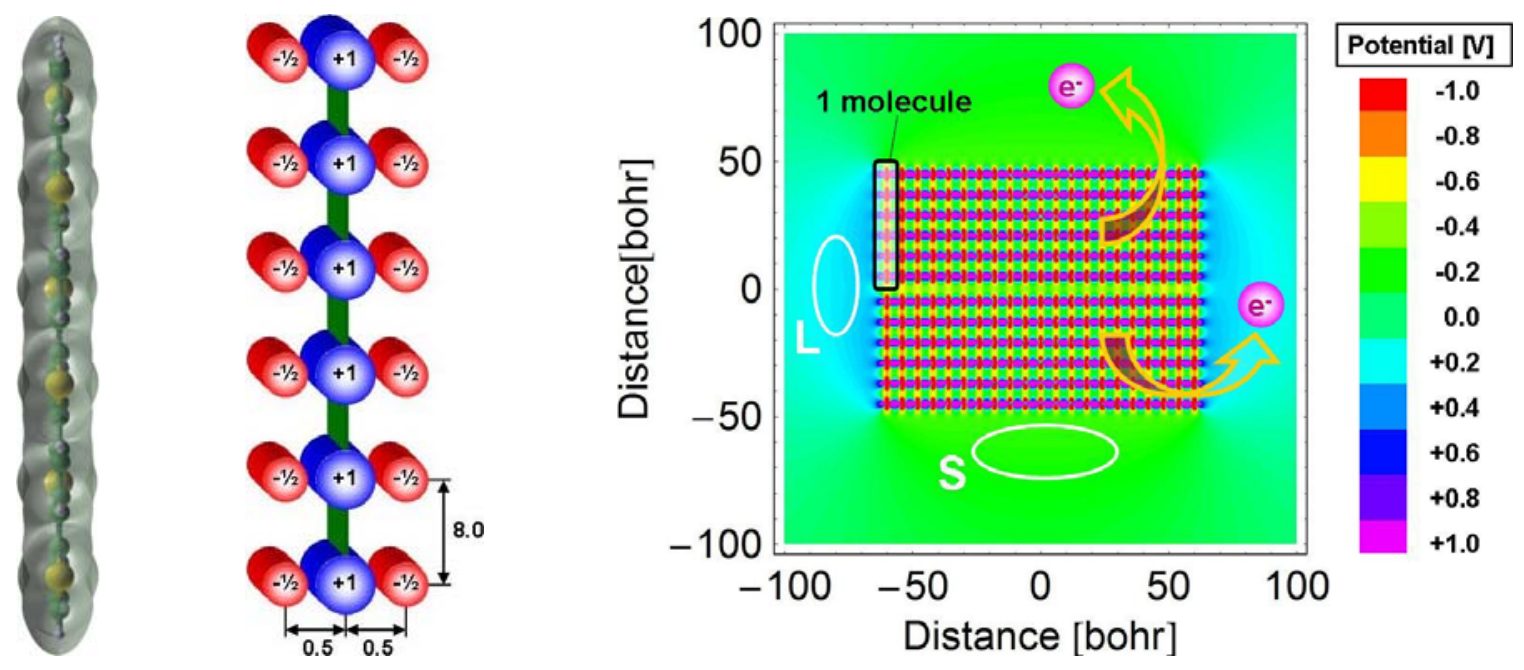

(a)

(b)

(c) 
S. Duhm et al., Nature Materials, accepted

Figure 6
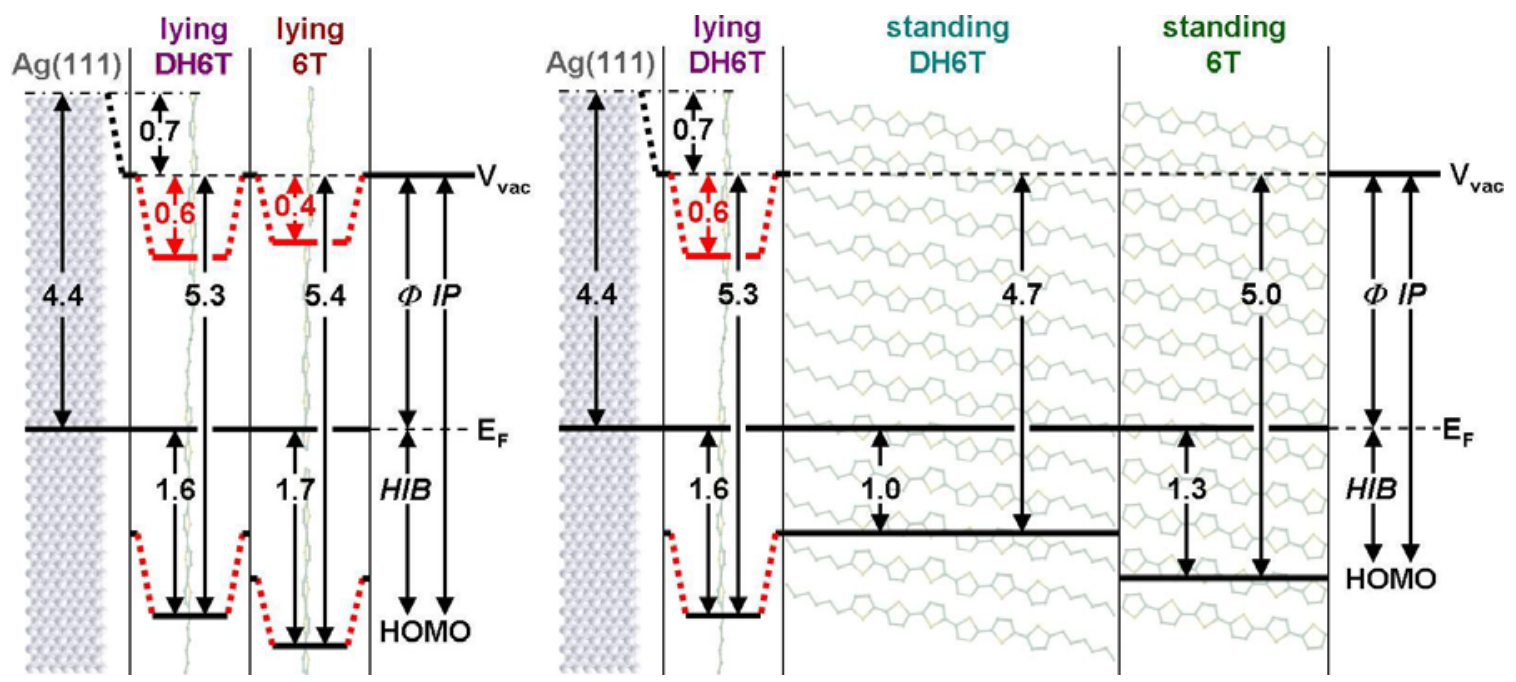

(a)

(b) 